\title{
Two-step vapor-crystal nucleation close below triple point
}

\author{
J. A. van Meel, ${ }^{1, a)}$ A. J. Page, ${ }^{2}$ R. P. Sear, ${ }^{2}$ and D. Frenkel ${ }^{1,3}$ \\ ${ }^{1}$ FOM Institute for Atomic and Molecular Physics, Kruislaan 407, 1098 SJ Amsterdam, The Netherlands \\ ${ }^{2}$ Department of Physics, University of Surrey, GU2 7XH, United Kingdom \\ ${ }^{3}$ Department of Chemistry, University of Cambridge, CB2 1EW, United Kingdom
}

(Received 17 June 2008; accepted 23 October 2008; published online 26 November 2008)

\begin{abstract}
We present the results of Monte Carlo simulations of crystal nucleation from the vapor phase. We studied the Lennard-Jones system at conditions close to, but below, the triple point. This system is expected to show surface melting. The nucleation pathway that we observe consists of two distinct steps. In the first step, a liquid droplet nucleates from the vapor. Its nucleation rate depends strongly on the vapor supersaturation. In the second step, the final crystal phase nucleates in the liquid droplet, provided that this liquid droplet exceeds a minimum size. Our simulations show that within a liquid droplet the crystal nucleation rate does not depend on the vapor supersaturation. In a recent independent study Chen et al. [J. Phys. Chem. B 112, 4069 (2008)] investigated the same phenomenon using umbrella sampling to compute free energy barriers and hence nucleation rates. We use a different numerical approach where we focus on computing the nucleation rates directly using forward-flux sampling. Our results agree with the findings of Chen et al. and both methods observe two-step nucleation. This finding indicates that this nucleation process can be described with a quasiequilibrium theory. Due to different cutoffs for the interaction potential the results cannot be compared quantitatively. (c) 2008 American Institute of Physics.
\end{abstract}

[DOI: $10.1063 / 1.3026364]$

\section{INTRODUCTION}

A first order phase transition starts with nucleation, if a free energy barrier separates the parent phase from the phase that is thermodynamically more stable. Nucleation from this metastable initial state will take place when a rare fluctuation allows the system to surmount the free energy barrier. Although the phase that forms during nucleation must be more stable than the parent phase, it is not necessarily the phase that is thermodynamically most stable. ${ }^{1}$ A metastable phase may nucleate if the transition to that phase is kinetically favored. A phase transition to a thermodynamically stable phase may therefore proceed via a two-step process. In the first nucleation event a metastable intermediate phase is formed; this then goes on to form the final phase via a second nucleation event.

There is experimental evidence that such a two-step nucleation scenario may be relevant to protein crystallization. For instance, lysozyme is sometimes found to separate into liquidlike aggregates before crystallization., ${ }^{2,3}$ This is at conditions at which lysozyme is only thermodynamically stable in the dilute fluid phase or in the crystal phase; the transition between a dilute and a concentrated liquid solution is located in a metastable region of the phase diagram where the solid phase is thermodynamically stable. Interestingly, crystal nucleation of these proteins appears to be near the metastable liquid-liquid coexistence curve. ${ }^{4,5}$ Simulations of enhanced crystal nucleation close to the metastable liquidliquid critical point show that the system first forms dense,

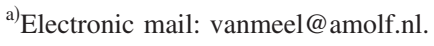

liquidlike clusters. ${ }^{4}$ Crystallites then form inside these liquid clusters. Several theoretical models provide a rationale for the behavior observed in the simulations. ${ }^{6-9}$

In the present paper we study the vapor of the LennardJones (LJ) system at temperatures below its triple point at chemical potentials where the crystal phase is the stable phase. Under these conditions, this system also acts as a simple model for "nanocolloids" in dilute solution. We find that, starting from a vapor, the phase transition does not occur directly to the crystal phase but via a liquid intermediate phase. The surface tension of the liquid-vapor interface is less than the surface free energy density of the crystal-vapor interface, making the free energy barrier to liquid droplet formation lower than to crystal formation. We expect that any phase transition, where an intermediate with a faster kinetic transition exists, will occur via a similar two-step process.

In a recent article, Chen et al. have used umbrella sampling to study two-step nucleation in the LJ system, ${ }^{10}$ where they applied classical nucleation theory (CNT) to estimate nucleation rates and critical nucleus sizes. In the present work, we present an independent study of the same phenomenon using forward-flux sampling (FFS). ${ }^{11,12}$ This technique primarily yields nucleation rates rather than free energy barriers, and allows to estimate the critical nucleus size directly from the simulation data. In addition, FFS includes the effect of kinetics which can seriously alter the observed nucleation phenomenon. ${ }^{13}$ Where a comparison can be made, our results are consistent with those of Ref. 10 (see Sec. V).

The remainder of the present paper is organized as follows. Section II deals with the simulation details and intro- 
duces the techniques that we have used. In Sec. III we present our results, and in Sec. IV we explain our result in the framework of CNT.

\section{SIMULATION DETAILS}

Throughout this work we report metropolis Monte Carlo (MC) simulations on a model system consisting of particles interacting through a truncated and shifted LJ pair potential.

$$
U(r)=\left\{\begin{array}{l}
U_{\mathrm{LJ}}(r)-U_{\mathrm{LJ}}\left(r_{c}\right), \quad r \leqslant r_{c} \\
0, \quad r>r_{c},
\end{array}\right.
$$

where the full (i.e., not truncated) LJ interaction is given by

$$
U_{\mathrm{LJ}}(r)=4 \epsilon\left[\left(\frac{\sigma}{r}\right)^{12}-\left(\frac{\sigma}{r}\right)^{6}\right] .
$$

Here, $\epsilon$ is the unit of energy, $\sigma$ is the unit of length, and $r_{c}$ the interaction cutoff distance. Note that the choice of interaction cutoff has a significant effect on the free energy of the system and on properties such as coexistence lines and surface tensions. ${ }^{14}$

In what follows, we use reduced units. We define the reduced distance as $r^{*}=r / \sigma$ and the reduced potential energy as $u^{*}=U \epsilon^{-1}$. All other reduced quantities (e.g., the pressure $P^{*}=P \sigma^{3} \epsilon^{-1}$, the density $\rho^{*}=\rho \sigma^{3}$, and the temperature $T^{*}$ $=k_{B} T \epsilon^{-1}$ ) follow. All quantities reported in this work are stated in these reduced units. Therefore, we omit the superscript $\operatorname{star}(*)$ from here on.

We performed all vapor-liquid simulations in the grandcanonical ensemble, where temperature, volume, and the chemical potential are kept constant, and the particle number is allowed to fluctuate. This ensures a constant vapor pressure and minimizes finite size effects. The resulting nucleation rates are presented per unit volume of the vapor phase.

Our liquid-solid simulations from a liquid droplet embedded in the vapor were performed both in the canonical and grand-canonical ensemble. The initial liquid droplet was taken both from grand-canonical vapor-liquid simulations, as well as from melting a fcc crystal at high temperatures, quenching it to $T=0.45$, and relaxing it for a long time. The results were unaffected by the preparation method.

Periodic boundary conditions were applied to all sides of the cubic simulation boxes. In the canonical ensemble simulations the box sizes were chosen to be $l^{3}=20^{3}$ for all but the largest droplet size, in which case the simulation was performed in a box of $l^{3}=30^{3}$. For all grand-canonical simulations the simulation box size was $l^{3}=44^{3}$. The trial move size is chosen to be $\Delta x=0.11$ in the canonical ensemble and $\Delta x$ $=0.2$ in the grand-canonical ensemble. In the grandcanonical ensemble each particle was moved on average 20 times before a insertion/removal move was performed.

To capture nucleation events, we applied the FFS scheme, a rare-event technique developed by Allen et al. ${ }^{11,12}$ FFS primarily yields nucleation rates. For the critical nucleus size an estimate is given by the order parameter value of the FFS interface at which the probability to reach the final state exceeds 0.5 . The error is then given by the FFS interface spacing. All nucleation rates and estimates for the critical cluster size were obtained by averaging over five indepen- dent FFS runs with at least 50 successful paths per interface. As the unit of time $\tau$ in our FFS simulations, we use a MC sweep, which corresponds to one trial displacement move per particle. For a given MC step size, $\tau$ can be related to the time in a molecular dynamics simulation by comparing the self-diffusion coefficient computed both from MC and molecular dynamics simulations. All nucleation rates are presented as the number of nuclei that form per unit volume and unit time, that is $[k]=\left[\tau^{-1} \sigma^{-3}\right]$. In addition, we also present the liquid-to-solid nucleation rates per droplet, $\left[k V_{D}\right]=\left[\tau^{-1}\right]$, where $V_{D}$ is the droplet volume. In the grand-canonical simulation, in which the droplet keeps growing, the initial droplet volume is used. To properly compare the nucleation rates between different ensembles, care has to be taken that the rates are expressed in the same unit of time. Furthermore, in the grand-canonical ensemble simulation the rate of particle insertion and deletion affects the density profile in the vicinity of a droplet surface, effectively changing the diffusion coefficient. However, small changes in our particle swap rates did not noticeably affect the nucleation rates. Therefore we neglected this effect throughout this work.

\section{A. Order parameters}

For the vapor-to-liquid nucleation we used an order parameter based on Stillinger's overlapping sphere criterion. ${ }^{15}$ It defines a particle to be in a high-density phase if it has at least one neighbor within a distance 1.5 corresponding roughly to the first minimum of the pair correlation function of a bulk liquid. Then a cluster analysis is performed on all high-density particles and the number of particles in the largest cluster is taken as the order parameter. Note that this order parameter does not distinguish between an ordered or disordered high-density phase, and therefore does not favor one phase (liquid or crystal) over the other. For a detailed description of this order parameter we refer the reader to Refs. 16 and 17.

As order parameter for the liquid-to-solid nucleation we applied the local bond-order parameter. ${ }^{18,19}$ This order parameter assigns each particle a 13-dimensional vector capturing its local environment,

$$
\left(q_{6}(i)\right)_{m}=\frac{1}{N_{b}(i)} \sum_{j} Y_{6 m}\left(\hat{r}_{i j}\right),
$$

where $Y_{6 m}$ denotes a sixth order spherical harmonic with components $m$ ranging from $-6 \leqslant m \leqslant 6 . N_{b}(i)$ is the number of nearest neighbors of particle $i$, and $\hat{r}_{i j}$ a unit vector connecting the centers of mass of particles $i$ and $j$. The sum is over all neighboring particles $j$ within a cutoff distance $r$ $=1.5$. In a second step the order parameter computes the dot product $\vec{q}_{6}(i) \cdot \vec{q}_{6}(j)$ between each particle $i$ and all its neighbors $j$, effectively comparing the particles' neighborhoods. If $\vec{q}_{6}(i) \cdot \vec{q}_{6}(j)$ exceeds a threshold of 0.65 , particles $i$ and $j$ are considered to form a "link." Only if a particle's total number of links $n(i)$ exceeds five links is it considered to be solidlike. In a final step Stillinger's criterion is applied to all solidlike particles to identify the number of particles in the largest solidlike cluster, which is used as order parameter. All parameters for this order parameter can be obtained by mini- 
TABLE I. For both the liquid (subscript $l$ ) and the fcc solid (subscript $s$ ) phase, the vapor supersaturation $S$ and the difference in chemical potential $\Delta \mu$ with respect to the vapor phase are presented as a function of the vapor pressure. The coexistence pressure for vapor-liquid is $P_{v l}^{\text {coex }}=4.28 \times 10^{-5}$ and for vapor-solid it is $P_{v s}^{\text {coex }}=2.28 \times 10^{-5}$. The difference in chemical potential between fcc solid and liquid is $\Delta \mu_{s l}=\mu_{s}-\mu_{l}=-0.29$.

\begin{tabular}{lrrrrrr}
\hline \hline$P_{v}\left(10^{-4}\right)$ & 1.0 & 2.0 & 3.0 & 4.0 & 5.0 & 6.0 \\
\hline$S_{s}$ & 4.39 & 8.77 & 13.16 & 17.54 & 21.93 & 26.28 \\
$S_{l}$ & 2.33 & 4.67 & 7.00 & 9.34 & 11.67 & 14.01 \\
$\Delta \mu_{s}$ & -0.67 & -0.98 & -1.16 & -1.29 & -1.39 & -1.47 \\
$\Delta \mu_{l}$ & -0.38 & -0.69 & -0.88 & -1.01 & -1.11 & -1.19 \\
\hline
\end{tabular}

mizing the overlap between distributions of an equilibrated bulk solid and a metastable bulk liquid at working conditions. Note that slight modification of these values affected neither the nucleation rate nor the nucleation pathway of our simulations. But it does affect the size of the clusters identified, and therefore the critical cluster size, too.

This local bond-order parameter was designed to detect nucleation in the bulk. In order to verify that nucleation at the droplet surface is not neglected, we also performed simulations with the modified local bond-order parameter used by Mendez-Villuendas and Bowles. ${ }^{20}$ This modification ensures that surface particles, too, can be identified as solidlike particles and are taken into account properly. However, compared to the unmodified order parameter no difference in the nucleation behavior was found.

\section{B. Simulation conditions}

In this study, the interaction cutoff distance was set to $r_{c}=2.5$. As the choice of cutoff distance affects the free energies of the system, we computed the free energies by using thermodynamic integration for the liquid ${ }^{21}$ and the Einstein crystal method for the fcc solid. ${ }^{22}$ Below the triple point the vapor behaves effectively like an ideal gas and its free energy can be computed analytically. We located the triple point to be at $T_{\mathrm{TP}}^{*} \approx 0.65$ and $P_{\mathrm{TP}}^{*} \approx 0.00271$.

Throughout this work the temperature was fixed at $T$ $=0.45\left(0.692 T_{\mathrm{TP}}\right)$. The vapor pressure was varied between $P_{v}=1 \times 10^{-4}$ and $P_{v}=5 \times 10^{-4}$. The bulk densities, obtained from NPT simulations with $N=2028$ particles, are $\rho_{L}$ $=0.905$ for the liquid and $\rho_{S}=0.989$ for the fcc solid. For both the liquid and solid phases, Table I lists the associated vapor supersaturation and the difference in chemical potential to the vapor phase. It is worth mentioning that at such low pressures the free energy of the high-density phases does not noticeably change with pressure. Therefore, once the coexistence pressure is known, the difference in chemical potential can be computed directly from the vapor supersaturation.

\section{RESULTS}

The aim of this work is to investigate the pathway for vapor-to-solid nucleation close to but below the triple point. Due to the existence of a large nucleation barrier this phenomenon is not accessible in a direct brute-force simulation. Even with FFS, a rare-event technique to overcome such
TABLE II. Vapor-liquid nucleation rates $k$ and the critical cluster size $n^{*}$ as a function of the vapor pressure $P_{V}$. All nucleation rates are obtained with FFS by using 50 paths for each interface and are averaged over five independent runs. The rates shown here are number of nuclei per unit volume and per MC cycle.

\begin{tabular}{ccc}
\hline \hline$P_{V}\left(10^{-4}\right)$ & $\ln k$ & $n^{*}$ \\
\hline 2.0 & $-117.3 \pm 0.6$ & $155 \pm 35$ \\
3.0 & $-75.4 \pm 1.2$ & $72 \pm 8$ \\
4.0 & $-56.6 \pm 0.8$ & $50 \pm 4$ \\
5.0 & $-47.6 \pm 0.9$ & $38 \pm 4$ \\
6.0 & $-39.4 \pm 0.7$ & $34 \pm 4$ \\
\hline
\end{tabular}

barriers, we could not observe direct vapor-to-solid nucleation. Instead, we find that a solid can be formed in a twostep process, where first a liquid droplet is nucleated from the vapor, and in a second nucleation event the crystal forms within the liquid droplet. We will discuss each nucleation step separately.

\section{A. Droplet nucleation from the vapor}

From the LJ phase diagram it is known that, at our conditions (see Sec. II), the crystal phase is the thermodynamically stable phase. ${ }^{23}$ In addition, our chemical potential calculations (see Table I) show that the liquid phase, also, has a lower free energy than the vapor phase. As a consequence, both a liquid and a solid can form. In order not to bias which high-density phase nucleates from the vapor, we use the local-density based order parameter discussed in Sec. II. This order parameter does not distinguish between ordered and disordered phases. In a canonical simulation the vapor is depleted of particles as the liquid droplet grows. For this reason we perform our simulations in the grand-canonical ensemble, which mimics conditions of constant vapor pressure.

Our simulations show only the nucleation of a liquid droplet. Analysis of the liquid droplets with a local bondorder parameter confirmed that the droplets did not contain any crystallites. The observed liquid clusters are on average compact spherical objects, which confirms previous results by ten Wolde and Frenkel ${ }^{16}$ and Wedekind and Reguera. ${ }^{17}$ The critical cluster size is approximated to be the FFS interface nearest to where the probability to grow to the final phase exceeds $P=0.5$. This will have an error equal to the gap between interfaces. A snapshot of a critical cluster is shown in Fig. 2(a). The compact spherical shape is clearly visible. The droplets continue growing, and for all clusters up to $N=3000$ particles, we did not observe any spontaneous crystallization.

The nucleation rates for the liquid are presented in Table II and in Fig. 1. The rate depends strongly on the vapor pressure. This is due to the fact that the free energy of the vapor is strongly affected by a slight change in pressure, whereas the free energy of the liquid is not.

This nucleation of a liquid phase, and the knowledge that the crystal phase has a lower free energy than the liquid phase, led us to investigate crystal nucleation within a liquid droplet. 


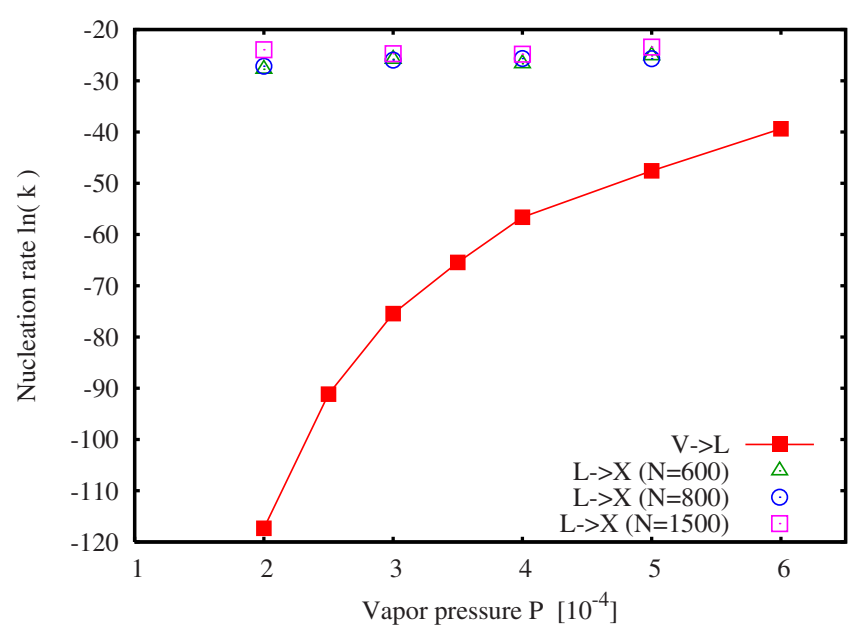

FIG. 1. (Color online) The logarithmic rate is plotted against the vapor pressure for the vapor-to-liquid (filled squares) and liquid-to-crystal (unfilled symbols) nucleation processes. The rates are expressed per unit volume of the vapor and liquid phases, respectively. Results for initial droplet sizes of 600 (triangles), 800 (circles), and 1500 (squares) are shown. The line through the filled symbols is a guide for the eye and errors are within the size of the symbols.

\section{B. Crystal nucleation within liquid droplets}

The nucleation of the crystal phase inside a liquid droplet was simulated in the canonical ensemble. We performed simulations to investigate how the size of the liquid droplet affects the nucleation of the crystal phase. The nucleation rates were obtained with the FFS ${ }^{11}$ technique, using the local bond-order parameter ${ }^{18,19}$ discussed in Sec. II. Simulations were performed on systems of size $N=448,612,700,850$, and 2028. The vast majority of particles formed part of the liquid droplet with, on average, less than seven particles forming the coexisting vapor. The vapor density, and hence the vapor pressure does not noticeably change as the crystal nucleates and grows inside the liquid droplet.

For all but the smallest system size with $N=448$, a stable or metastable crystallite was nucleated. Nucleation occurs in the core of the droplet and not at the surface like the freezing of gold nanoclusters. ${ }^{20}$ In the LJ system close below its triple point the crystal-vapor interface has a higher free energy cost than the liquid-vapor interface and surface melting is expected. ${ }^{24}$ This phenomenon can be seen in Fig. 2(c), a
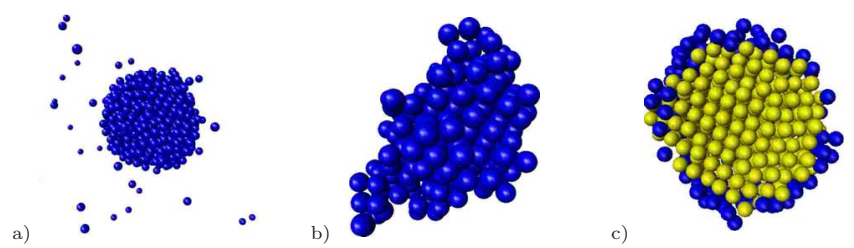

FIG. 2. (Color online) (a) Snapshot of a liquid cluster from a grandcanonical simulation clearly showing its compact spherical shape. (b) A critical crystal cluster from a canonical simulation containing 2028 particles. Only the crystal particles are shown in this snapshot. This crystal cluster contains 192 crystal particles. Snapshots of the critical cluster from simulations containing 700 and 850 particles are indistinguishable from the cluster shown here. (c) Cross section of a crystal cluster in a system size of 850 particles. Light particles are crystalline, dark are liquid. The crystal is coated in a liquidlike layer. This layer is approximately one particle in thickness. In snapshots (b) and (c) the crystal particles were characterized by bond-order parameters as detailed in Sec. II.
TABLE III. For a liquid droplet embedded in a vapor, this table shows the logarithmic rate for liquid-to-crystal nucleation and the critical cluster size as a function of the number of particles $N_{D}$ in the liquid droplet. All simulations were performed in the canonical ensemble. The rates are expressed per droplet $\ln \left(k V_{D}\right)$ and per unit volume $\ln (k)$, where $V_{D} \approx N_{D} / \rho_{L}$ is the droplet volume. The unit of time is a Monte Carlo cycle. The critical cluster results have an error of \pm 10 .

\begin{tabular}{cccc}
\hline \hline No. of particles $N_{D}$ & $\ln \left(k V_{D}\right)$ & $\ln (k)$ & $n^{*}$ \\
\hline 448 & $\ldots$ & $\ldots$ & $\ldots$ \\
612 & $-23.7 \pm 1.5$ & $-30.2 \pm 1.5$ & 180 \\
700 & $-22.1 \pm 1.0$ & $-28.8 \pm 1.0$ & 190 \\
850 & $-20.7 \pm 1.0$ & $-27.6 \pm 2.0$ & 180 \\
2028 & $-21.1 \pm 1.6$ & $-28.8 \pm 1.6$ & 190 \\
\hline \hline
\end{tabular}

snapshot of a crystallized cluster. Notice that a disordered layer of approximately one particle thickness separates the crystal and vapor phases. When we continue the simulations after crystal nucleation is complete, we find that the particles in this disordered layer diffuse around the surface of the crystal cluster. Thus the monolayer is liquidlike.

In small droplets of 448 particles we find that all crystallites that form are unstable and quickly melt. The absence of a stable or metastable crystallites suggests that the crystal phase for these small droplets is unstable due to the high free energy cost of the liquid-crystal interface. For the droplets that crystallized, the natural logarithms of the nucleation rates are shown in Table III. The nucleation rate per droplet, directly provided by our FFS simulations, is an extensive property; we therefore expressed the rates per unit volume of the liquid, too. The droplet volume is estimated by $V_{D}$ $\approx N_{D} / \rho_{L}$, where $N_{D}$ are the number of particles in the droplet, and $\rho_{L}$ is the liquid density at coexistence.

Further analysis of our FFS data shows that the critical cluster occurs within a range of cluster sizes from 180 to 200 particles. This range is the same for all system sizes consisting of 700 to 2028 particles. A snapshot of a critical cluster is shown in Fig. 2(b).

\section{Crystallization in the grand-canonical ensemble}

To test out calculations of the crystal nucleation rates in the canonical ensemble, we also performed simulations in the grand-canonical ensemble. Apart from the ensemble both simulation methods were identical. As starting configurations we used postcritical liquid droplets with $N=600, N=800$, and $N=1500$ particles.

Figure 1 shows logarithmic nucleation rates for the vapor-liquid and liquid-crystal against vapor pressure. Nucleation inside the liquid droplet is not affected significantly by the vapor pressure. The pressure inside the droplet is approximated to be

$$
P_{\text {droplet }}=P_{\text {vapor }}+P_{\text {Laplace }},
$$

where

$$
P_{\text {Laplace }}=\frac{2 \gamma_{l v}}{R} .
$$

Using the virial pressure tensor we computed that $\gamma_{l v}=1.07$. Taking the droplet in Fig. 2(b) as an example, the radius is 
TABLE IV. For a liquid droplet embedded in a vapor the logarithmic rate for liquid-to-crystal nucleation and the critical cluster size is shown as a function of vapor pressure. Results for different initial droplet sizes $N_{D}$ $=600,800,1500$ are presented both as rates per droplet $\ln \left(k_{N} V_{N}\right)$ and as rates per unit volume $\ln \left(k_{N}\right)$, where $V_{N} \approx N_{D} / \rho_{L}$ is the droplet volume. All simulations were performed in the grand-canonical ensemble, therefore the particle numbers $N_{D}$ and the associated droplet volume $V_{D}$ are those at the start of the simulation. The error for the critical cluster size is \pm 20 .

\begin{tabular}{lcccc}
\hline \hline \multicolumn{1}{c}{$P_{V}\left(10^{-4}\right)$} & 2.0 & 3.0 & 4.0 & 5.0 \\
\hline $\ln \left(k_{600} V_{600}\right)$ & $-21.4 \pm 2.0$ & $-19.4 \pm 0.9$ & $-20.3 \pm 1.5$ & $-18.7 \pm 1.4$ \\
$\ln \left(k_{800} V_{800}\right)$ & $-20.5 \pm 1.8$ & $-18.3 \pm 1.0$ & $-19.0 \pm 0.7$ & $-19.0 \pm 2.5$ \\
$\ln \left(k_{1500} V_{1500}\right)$ & $-16.6 \pm 1.5$ & $-17.4 \pm 2.3$ & $-17.5 \pm 2.1$ & $-16.2 \pm 2.5$ \\
$\ln \left(k_{600}\right)$ & $-27.9 \pm 2.0$ & $-25.9 \pm 0.9$ & $-26.8 \pm 1.5$ & $-25.2 \pm 1.4$ \\
$\ln \left(k_{800}\right)$ & $-27.3 \pm 1.8$ & $-25.1 \pm 1.0$ & $-25.8 \pm 0.7$ & $-25.8 \pm 2.5$ \\
$\ln \left(k_{1500}\right)$ & $-24.0 \pm 1.5$ & $-24.8 \pm 2.3$ & $-24.9 \pm 2.1$ & $-23.6 \pm 2.5$ \\
$n_{(600)}^{*}$ & 180 & 180 & 180 & 190 \\
$n_{(800)}^{*}$ & 180 & 180 & 170 & 170 \\
$n_{(1500)}^{*}$ & 170 & 160 & 180 & 170 \\
\hline
\end{tabular}

approximately $R \approx 5$, giving a Laplace pressure $P_{\text {Laplace }}$ $\approx 0.4$. So although an increase in the vapor pressure does increase the pressure inside the droplet, we see this increase is small compared to the Laplace pressure, $10^{-4} \ll 0.4$. The liquid and crystal phases are dense hence any increase in pressure of this order (i.e., $10^{-4}$ ) does not notably change the chemical potential. This is in contrast to the vapor phase where small changes in the vapor pressure cause large changes in the chemical potential and hence in the free energy barrier.

In the grand-canonical ensemble, the liquid droplets keep growing during the FFS simulations. At a pressure of $3 \times 10^{-4}$ the droplet initially containing 1500 particles grew to $6581 \pm 3349$ particles whereas the $N=600$ droplet only grew to $1875 \pm 323$ particles. The larger increase in particle number of the $N=1500$ droplet is due to the larger surface area than the smaller droplets. We expect this increase in the droplet size to be the cause of the systematically higher rates for the $N=1500$ droplet. We point out that due to the droplet growth the droplet volume is not a well-defined quantity. For the nucleation rate per unit volume we therefore use the initial droplet volume for the normalization.

However, the observed nucleation rates (see Table IV) are comparable to the rates obtained from canonical simulations (see Table III) and both methods yield the same value for the critical nucleus. Nucleation of the liquid phase from the vapor is the rate-limiting step. At a vapor pressure of $P_{V} \approx 10^{-3}$ the nucleation rates of the liquid and crystal phases are of the same order of magnitude. We expect that, as the pressure increases, the nucleation of the crystal phase inside the liquid droplet will become the rate-limiting step.

Note that for a proper comparison of canonical and grand-canonical nucleation rates both need to be expressed in the same unit of time $\tau$ (see Sec. II for details). Therefore, the comparison remains qualitative.

\section{Direct crystal nucleation from the vapor}

Between the vapor-solid and metastable vapor-liquid coexistence curves $\left(P_{v l}=2.28 \times 10^{-5}<P<P_{v s}=4.28 \times 10^{-5}\right)$, the liquid phase has a higher free energy than the vapor phase. Here we would expect direct nucleation to the crystal phase instead of the two-step nucleation detailed in this paper. At these conditions we could not get our system to crystallize at all. No direct vapor-crystal nucleation was observed in any of our simulations. This illustrates the dramatic effect of the intermediate metastable liquid on the crystal nucleation rate.

\section{CNT}

Since the direct nucleation of the crystal phase from the vapor cannot be seen in simulation we decided to estimate the nucleation rate via $\mathrm{CNT}^{25}$ This theory assumes that the crystal nuclei are perfectly spherical and incompressible. The resulting free energy barrier is then

$$
\Delta G^{*}=\frac{16 \pi}{3} \frac{\gamma_{v s}^{3}}{\rho_{s}^{2}\left|\Delta \mu_{s}\right|^{2}},
$$

where $\Delta \mu_{s}$ is the difference in chemical potential between the vapor and the solid from Table I. The vapor-crystal surface tension $\gamma_{v s}$ is unknown and must be estimated. As lower bound we use the planar vapor-liquid surface tension $\gamma_{v l}$, which can be computed from the virial pressure tensor obtained from simulation. ${ }^{21} \mathrm{We}$ find $\gamma_{v l}=1.07$. Note that the surface tension depends strongly on the interaction cutoff distance, and for $r_{c}=2.5$ it significantly deviates from the untruncated LJ system. Using $\gamma_{v l}=1.07, \Delta \mu_{s}=-0.29$ and $\rho_{s}$ $=0.989$ we obtain $\Delta G^{*}=250$ for a lower bound for the free energy barrier. The resulting nucleation rate is essentially zero, which explains why no direct crystal nucleation was observed.

Using the same value for the surface tension and the data from Table I the simulation data can be compared to CNT. In literature it is common to compare nucleation free energy barriers rather than nucleation rates. As FFS yields nucleation rates directly, we approximate the barrier height using the CNT rate expression to obtain

$$
\Delta G_{\mathrm{FFS}}^{*}=-k_{B} T \ln \left(k_{\mathrm{FFS}} / J_{0}\right),
$$

where $J_{0}$ is the kinetic prefactor from the CNT rate expression. Note that, in contrast to the work of Chen et al., errors in the CNT kinetic prefactor will influence the estimate of the barrier height computed by FFS. But since these errors have logarithmic dependence we considered them negligible. The kinetic prefactor $J_{0}$ can be approximated by ${ }^{26}$

$$
J_{0} \approx Z \rho_{v}^{2} D R^{*},
$$

where $D$ is the self-diffusion coefficient and

$$
Z=\sqrt{\frac{|\Delta \mu|}{6 \pi T N^{*}}}
$$

is the Zeldovich factor. ${ }^{27}$ Note that the above expression for $J_{0}$ assumes that the dynamics of droplet nucleation and growth is diffusive. Such a description is compatible with the use of MC simulations to model the dynamics of a diffusive nanocolloidal system. For a dilute vapor the self-diffusion coefficient can be estimated from $D=\Delta x^{2} / \tau=0.04$, the MC 

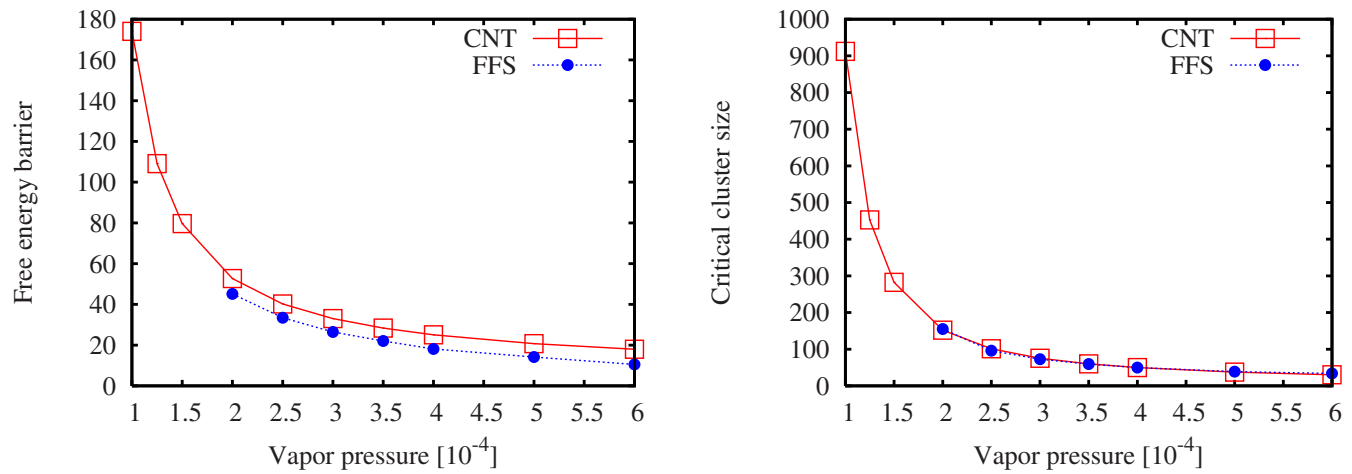

FIG. 3. (Color online) Comparison of CNT predictions versus the simulation results for the vapor-to-liquid nucleation. Both the nucleation barrier height $\Delta G^{*}$ (left) and the critical cluster size $n^{*}$ (right) are plotted as a function of vapor pressure. The constant offset in barrier height is attributed to the accumulated error in the CNT description for very small clusters (Ref. 28).

step size squared per MC cycle. The CNT critical droplet radius $R^{*}$ is given by

$$
R^{*}=\frac{2 \gamma_{v l}}{\rho_{l}\left|\Delta \mu_{l}\right|}
$$

For the conditions that apply to the present simulations, we find that $J_{0}$ varies between $J_{0} \approx 2.2 \times 10^{-11}$ and $1.6 \times 10^{-9}$ for pressures ranging between $P_{v}=1.0 \times 10^{-4}$ and $5.0 \times 10^{-4}$. The results are shown in Fig. 3. The functional form of the CNT barrier is in good agreement with our simulation results, but a constant offset is observed. This is consistent with earlier work on vapor-liquid nucleation. ${ }^{10,16,28,29}$ But even though many offset values are reported in literature, the applied interaction cutoffs, temperatures and supersaturations vary strongly making a direct comparison difficult. Since the precise composition of the barrier height offset to CNT is still debated ${ }^{28-32}$ we only compare the total offset $D(T)$. From our simulations we obtain $D(T)=7.5$, which is in good agreement with the results from ten Wolde and Frenkel, ${ }^{16}$ $D(T)=5.3$, that were obtained with the same truncation and shift, but at a slightly higher temperature of $T=0.741$. These values are lower than those reported by McGraw et al. ${ }^{29}$ $(D(T=0.8) \approx 19-20), \quad$ Merikanto et $a .^{33} \quad(D(T=0.7)$ $\approx 18-19)$, and Chen et al. ${ }^{34}(D(T=0.6) \approx 22$ to $D(T=0.45)$
₹27), who used the full LJ potential. This deviation is not unexpected and can be attributed to the effect of the interaction potential's long-range tail on the surface tension $\gamma$ and, even more, on its curvature correction $B(T)$.

In order to use CNT to analyze the nucleation of crystallites inside liquid droplets, we use a simplified model: first, we assume that the crystallite and liquid droplet are perfect spheres. Second, the crystal is assumed to grow from the center of the liquid droplet (i.e., we assume bulk rather than surface nucleation). Furthermore, as suggested by our simulations, we assume that the droplet is always covered with at least a monolayer of liquid. Finally, we postulate that the surface free energies are independent of each other no matter how close the crystal-liquid and liquid-vapor interfaces are. A diagram of this crude but effective model is shown in Fig. 4(a). Using this model, the free energy barrier for crystal nucleation can be approximated by Eq. (4) where $\Delta \mu_{s}$ is exchanged with $\Delta \mu_{l x}$, the difference between the bulk liquid and crystal chemical potentials (from Table I), and $\gamma_{v s}$ is exchanged with $\gamma_{l x}$, the liquid-crystal surface tension. As an estimate we take $\gamma_{l x}=0.347$. This value is the planar surface tension for the (111) crystal plane calculated by Davidchack and Laird ${ }^{35}$ at the triple point. They used a truncated and force shifted LJ potential at the same cutoff as our simu-
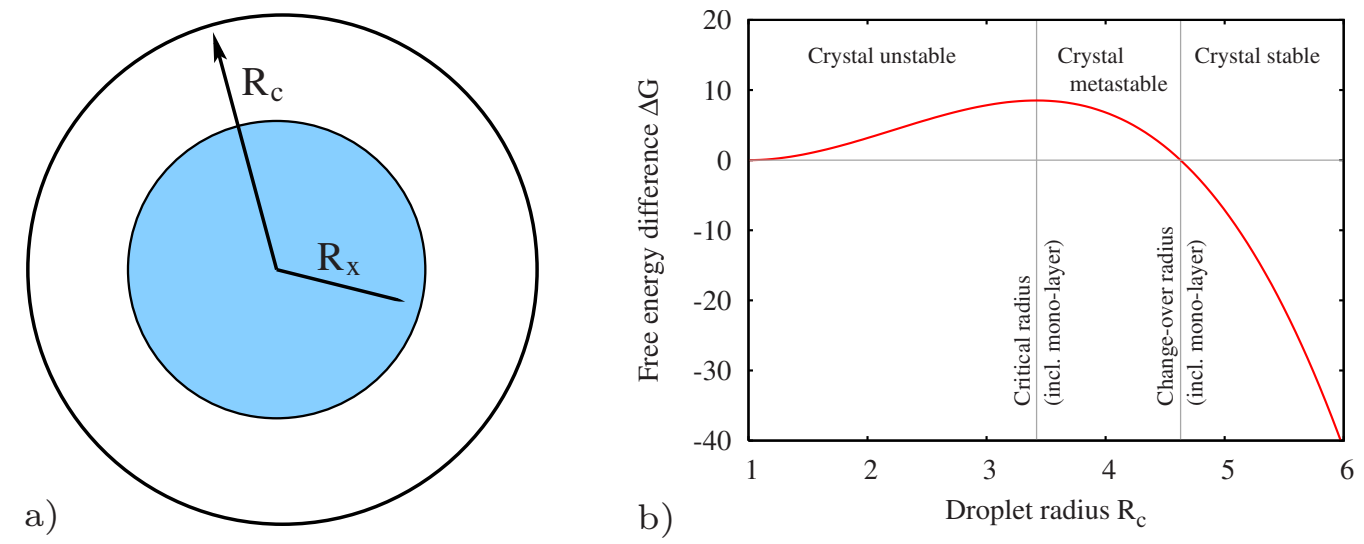

FIG. 4. (Color online) (a) Diagram of the assumed geometry of a crystal cluster growing in a liquid droplet. The size of the crystal cluster $R_{x}$ is always at least one less than the size of the liquid droplet it grows within, $R_{c}$, to account for the liquidlike monolayer. (b) Free energy difference between a purely liquid droplet and the crystal state, a droplet containing a crystal of radius $R_{x}=R_{c}-1$, the largest crystal the droplet can support. Depending on the droplet radius $R_{c}$, the crystal state of the droplet will either be unstable, metastable, or stable with respect to the purely liquid droplet. Note the droplet has a fixed radius and we do not refer to the bulk phase stability. 
lations but as we are working under the triple point we expect the actual value to be lower than this. This simple model gives an estimated free energy barrier of $\Delta G_{l x}^{*}=-8.5\left(\Delta G_{l x}^{*} / k T=-18.9\right)$.

Further, assuming that the surface tension does not depend on the radius of curvature of the droplet (a rather drastic assumption ${ }^{36}$ ), all crystallites have the same free energy curve. Note that, unlike for bulk systems, this free energy curve is terminated at $R_{x}=R_{c}-1$, the droplet radius minus the liquid monolayer. The droplet therefore has two distinct states: the liquid state referring to a purely liquid droplet, and the crystalline state referring to the droplet containing a crystal of maximum size $\left(R_{x}^{(\max )}=R_{c}-1\right)$.

The free energy difference between these two states is shown in Fig. 4(b) as a function of the droplet radius $R_{c}$. We now introduce the change-over radius $R_{x}^{c o}$, which defines the point where both states, the liquid state and the crystalline state, have the same free energy. Note that this is not exactly the radius where there is an equal probability to find droplet on the liquid or crystalline side of the nucleation barrier as the ratio of these probabilities also depends on the ratio of the "volume" in the free energy landscape on either side of the barrier. However, if these (logarithmic) phase-space volume corrections are small, $R_{x}^{c o}$ provides a good approximation of the point where crystal and liquid are equally likely.

We can now identify three different classes for the droplet radius where the crystalline state is either (a) unstable, (b) metastable, or (c) stable with respect to the droplet in the liquid state [see Fig. 4(b)].

(a) Unstable: $R_{c} \leqslant R_{x}^{*}+1$, where $R^{*}$ is the critical radius of a crystal nucleus in a bulk liquid. If the droplet is smaller than the crystal critical radius any crystal clusters formed are unstable and will only be observed as rare fluctuations.

(b) Metastable: $R_{x}^{*}+1<R_{c} \leqslant R_{x}^{c o}+1$. Above the critical radius there is a free energy barrier associated with the crystalline state returning to the liquid state. The droplet has a small probability of being in the crystalline state but, as remelting is an activated process, the lifetime of these metastable crystalline states may be long. Since the crystalline state has a higher free energy the droplet spends the majority of its time in the liquid state. This "metastable" regime ends for $R_{c}=R_{x}^{c o}+1$ where the crystalline state becomes more stable than the liquid.

(c) Stable: $R_{c}>R_{x}^{c o}+1$. The change-over radius determines the minimum size for the crystalline state to be more stable than the liquid. In this class the liquid state is now metastable.

The crystallites inside the droplets follow the same free energy curve and therefore the free energy barrier is independent of the size of the droplet. We observe this feature in our simulation results where the liquid-crystal nucleation rates are to a good approximation independent of droplet size. A similar scenario is found in the simulation of explosive melting of polymer crystallites. ${ }^{37}$

CNT predicts the critical radius and the change-over ra- dius to be $R_{x}^{*}[\mathrm{CNT}]=2.4$ and $R_{x}^{c o}[\mathrm{CNT}]=3.6$, respectively. A comparison with the simulation results remains qualitative, as the characterization of the crystal phase, and therefore the critical cluster size, depends on the definition of order parameter used for analysis (see Sec. II). Analyzing our FFS data yields a critical cluster size of $n_{\mathrm{sim}}^{*} \approx 190$ particles which corresponds to a radius of $R_{x}^{*}[\mathrm{SIM}] \approx 3.6$ (without liquid monolayer). This critical radius obtained from FFS is close to the droplet size $R_{c}=4.8$ [see Fig. 4(a)] found for a $N$ $=448$ system size. At this proximity to the critical radius it is not surprising that we find the droplet to be stable in the liquid state. For droplets of size $N \approx 600$, the crystalline state is expected to be metastable, and for $N \approx 700$ both states should be observable. Droplets exceeding $N \approx 800$ are expected to be stable in the crystal phase.

\section{DISCUSSION}

This paper details the nucleation pathway from a metastable vapor just below the triple-point temperature to the crystal phase. This process is relevant for the fabrication of nanocrystals, and it can also act as a model for crystallization of nanocolloids from a dilute solution.

At these conditions, we find that the vapor-to-crystal nucleation occurs in two stages. First, an intermediate liquid phase is nucleated rather than the thermodynamically most stable crystal phase. The final crystal phase then nucleates in the liquid phase in a second independent step. This two-stage process suggests that both the vapor-liquid and liquid-crystal free energy barriers are lower than that of a direct nucleation of the crystal from the vapor. ${ }^{1,38}$ This is supported by both an analysis of surface free energies and a comparison with CNT. Direct nucleation of the crystal is not observed and a CNT estimate of this nucleation rate is essentially zero.

A recent publication by Chen et al. came to conclusions similar to ours, on the basis of a different numerical approach. ${ }^{10}$ Chen et al. obtained free energy curves from aggregation-volume-bias MC simulations with umbrella sampling. As the reaction coordinate for the crystal phase they used the global bond-order parameter, which detects the overall crystallinity of the liquid cluster. ${ }^{39}$ Both nucleation rates and critical cluster size were estimated using CNT. In contrast, we focus on computing nucleation rates directly rather than free energy barriers by using the FFS technique, which can be used both for equilibrium and nonequilibrium processes. ${ }^{12}$ As order parameter the size of the largest crystal cluster in the liquid droplet was used. The critical nuclei were then obtained directly from analysis of our FFS data.

To compare with the work in Ref. 10 we must translate nucleation rates in free energy barriers. The fact that we obtain reasonable agreement with Ref. 10 suggests that CNT works rather well for this system, and that this process can be described with a quasiequilibrium theory. In addition, note that both umbrella sampling and FFS essentially yields the same results for the LJ system. As reported by Sanz et al. this need not to be true for every system. ${ }^{13}$

A quantitative comparison between the present simulations and those of Ref. 10 is unfortunately not possible as the studies used different cutoffs for the LJ potential. The change 
in cutoff affects the chemical potentials for both the liquid and crystal phases, and it also changes the surface tensions. ${ }^{14}$ As both properties have a large impact on the resulting nucleation free energy barrier, the comparison remains qualitative.

To conclude, this work details the process of crystal nucleation from the vapor phase. The two-stage nucleation found is potentially a very common phenomenon that could occur in fields as diverse as protein crystallization and ice formation.

\section{ACKNOWLEDGMENTS}

J.A.v.M. and A.J.P. contributed equally to this work. A.J.P. would like to thank EPSRC for the award of a studentship. The work of the FOM Institute is part of the research program of FOM and is made possible by financial support from the Netherlands Organization for Scientific Research (NWO). The authors thank P. Charbonneau for a careful reading of the manuscript.

${ }^{1}$ W. Ostwald, Z. Phys. Chem. 22, 289 (1897).

${ }^{2}$ M. Muschol and F. Rosenberger, J. Chem. Phys. 107, 1953 (1997).

${ }^{3}$ Y. G. Kuznetsov, A. J. Malkin, and A. McPherson, J. Cryst. Growth 232 30 (2001).

${ }^{4}$ P. R. ten Wolde and D. Frenkel, Science 277, 1975 (1997).

${ }^{5}$ O. Galkin and P. G. Vekilov, Proc. Natl. Acad. Sci. U.S.A. 97, 6277 (2000).

${ }^{6}$ V. Talanquer and D. W. Oxtoby, J. Chem. Phys. 109, 223 (1998).

${ }^{7}$ R. P. Sear, J. Chem. Phys. 114, 3170 (2001).

${ }^{8}$ G. Nicolis and C. Nicolis, Physica A 323, 139 (2003).

${ }^{9}$ J. F. Lutsko and G. Nicolis, Phys. Rev. Lett. 96, 046102 (2006).

${ }^{10}$ B. Chen, H. Kim, S. J. Keasler, and R. B. Nellas, J. Phys. Chem. B 112, 4067 (2008)
${ }^{11}$ R. J. Allen, P. B. Warren, and P. R. ten Wolde, Phys. Rev. Lett. 94, 018104 (2005).

${ }^{12}$ R. J. Allen, D. Frenkel, and P. R. ten Wolde, J. Chem. Phys. 124, 024102 (2006).

${ }^{13}$ E. Sanz, C. Valeriani, D. Frenkel, and M. Dijkstra, Phys. Rev. Lett. 99, 055501 (2007).

${ }^{14}$ V. G. Baidakov, G. G. Chernykh, and S. P. Protsenko, Chem. Phys. Lett. 321, 315 (2000).

${ }^{15}$ F. H. Stillinger, J. Chem. Phys. 38, 1486 (1963).

${ }^{16}$ P. R. ten Wolde and D. Frenkel, J. Chem. Phys. 109, 9901 (1998).

${ }^{17}$ J. Wedekind and D. Reguera, J. Chem. Phys. 127, 154516 (2007).

${ }^{18}$ P. R. ten Wolde, M. Ruiz-Montero, and D. Frenkel, Nature (London) 409, 1020 (2001).

${ }^{19}$ S. Auer and D. Frenkel, Faraday Discuss. 104, 93 (1996).

${ }^{20}$ E. Mendez-Villuendas and R. K. Bowles, Phys. Rev. Lett. 98, 185503 (2007).

${ }^{21}$ D. Frenkel and B. Smit, Understanding Molecular Simulation (Academic, New York, 2002).

${ }^{22}$ D. Frenkel and A. J. C. Ladd, J. Chem. Phys. 81, 3188 (1984).

${ }^{23}$ M. A. van der Hoef, J. Chem. Phys. 113, 8142 (2000).

${ }^{24}$ R. Ohnesorge, H. Löwen, and H. Wagner, Phys. Rev. E 50, 4801 (1994).

${ }^{25}$ D. Turnbull and J. C. Fisher, J. Chem. Phys. 17, 71 (1949).

${ }^{26}$ R. P. Sear, J. Phys.: Condens. Matter 19, 033101 (2007).

${ }^{27}$ J. Zeldovich, J. Exp. Theor. Phys. 12, 525 (1942)

${ }^{28}$ J. Merikanto, E. Zapadinsky, A. Lauri, and H. Vehkamäki, Phys. Rev. Lett. 98, 145702 (2007).

${ }^{29}$ R. McGraw and A. Laaksonen, J. Chem. Phys. 106, 5284 (1997).

${ }^{30}$ S. L. Girshick and C.-P. Chiu, J. Chem. Phys. 93, 1273 (1990).

${ }^{31}$ A. Laaksonen, I. J. Ford, and M. Kulmala, Phys. Rev. E 49, 5517 (1994).

${ }^{32}$ M. Horsch, J. Vrabec, and H. Hasse, Phys. Rev. E 78, 011603 (2008).

${ }^{33}$ Only $B(T)$ and $\gamma$ were provided in Ref. 28. For the estimation of $D(T)$ $=B(T)+A_{1} \gamma-|\Delta \mu|$ we assumed $\rho_{L} \approx 0.84$ and $\Delta \mu=-1.0$.

${ }^{34}$ Data extracted from the graphical representation in Ref. 10.

${ }^{35}$ R. L. Davidchack and B. B. Laird, J. Chem. Phys. 118, 7651 (2003).

${ }^{36}$ T. Zykova-Timan, C. Valeriani, E. Sanz, D. Frenkel, and E. Tosatti, Phys. Rev. Lett. 100, 036103 (2008).

${ }^{37}$ W. Hu, D. Frenkel, and V. B. F. Mathot, J. Chem. Phys. 118, 3455 (2003).

${ }^{38}$ I. Stranski and D. Totomanov, Z. Phys. Chem. 163, 399 (1933).

${ }^{39}$ J. S. van Duijneveldt and D. Frenkel, J. Chem. Phys. 96, 4655 (1992). 